\title{
TOWARDS DESIGN OF HIGH SPIN METAL-FREE MATERIALS
}

\author{
NGUYEN VAN THANH \\ Faculty of Physics, VNU University of Science, \\ 334 Nguyen Trai, Thanh Xuan, Hanoi, Vietnam \\ NGUYEN ANH TUAN \\ Faculty of Physics, VNU University of Science, \\ 334 Nguyen Trai, Thanh Xuan, Hanoi, Vietnam \\ and \\ Japan Advanced Institute of Science and Technology, \\ 1-1, Asahidai, Nomi, Ishikawa, 923-1292 Japan \\ Received 19 Decmenber 2013 \\ Accepted for publication 31 December 2013
}

\begin{abstract}
In this paper, in order to explore high-spin carbon-based magnetic materials with strong ferromagnetic coupling, geometric structure, electronic structure and magnetic properties of alternating stacks of $\pi$-radical-halogenated-hydrocarbons and diamagnetic molecules have been investigated based on density-functional theory with dispersion correction. These alternating stacks are predicted to avoid the typical antiferromagnetic spin-exchange of indentical face-to-face radicals via spin polarization of a diamagnetic molecule in between. Our results show that $\pi$-radicalhalogenated-hydrocarbons like perchlorophenalenyl $\left(C_{13} C l_{9}\right)$ is strong ferromagnetic coupling if alternatingly stacked with aromatics like fluorinated coronene $\left(C_{24} F_{12}\right)$ or coronene $\left(C_{24} H_{12}\right)$, while fluorinated perinaphthenyl $\left(C_{13} F_{9}\right)$ and perinaphthenyl $\left(C_{13} H_{9}\right)$ are not an equally good choice. The role of ligand configuration in determining exchange coupling in stacks is discussed. These results would give some hints for designing new high-spin carbon-based ferromagnetic materials.
\end{abstract}

\section{INTRODUCTION}

Carbon is not only known as a biocompatible element, but also many advanced carbon-based materials with special functional and properties were synthesized, such as carbon nanotubes, fullerences, graphene. Moreover, in the past twenty years metal-free magnets made from carbon-based compounds have been discovered and, more recently, there have been reports on the observation of magnetism in carbon [1-9]. Metal-free carbon structures exhibiting magnetic ordering represent a new class of materials and open a novel field of research that may lead to many new technologies [1,2]. No physical law prohibits magnetic ordering in the structures containing only light elements like carbon. Bulk crystalline graphite is a strong diamagnet, with the magnetic susceptibility second only to superconductors. However, graphite containing certain defects can exhibit spontaneous magnetization $[2-5,7]$. Previous experimental studies also showed that long-range magnetic order in carbon-based materials can exist above room temperature $[2-5,7]$. It is greatly 
surprised that magnetism in carbon-based materials is determined by s- and p-electrons $[2,9]$. However, the origin of magnetic order in pure carbon in only poorly understood $[2,4,5]$. Studying on the mechanism for localized magnetic moments and long-range magnetic order in carbon-based magnetic materials is major problems for development of these materials. The large and growing number of publications and patents in ferromagnetism of carbon-based magnetic materials have been reported [1-8]. In early 2000s, carbon-based materials with long-range magnetic order at room temperature have been explored [2]. However, the existence of room temperature carbon-based ferromagnets were till the accident discovery $[2-5,7]$. Moreover, their saturation magnetization is usually small $\mathrm{M}_{S} \approx$ $0.1-1 \mathrm{emu} / \mathrm{g}[2]$. There is only one report about a carbon-ion-implanted highly oriented pyrolytic graphite having $\mathrm{M}_{S}=9.3 \mathrm{emu} / \mathrm{g}$ up to now [5]. How to produce carbon-based magnetic materials with long-range ferromagnetic at high temperature and significant magnetization is still a big challenge for scientists.

In theoretical study, there are several models of carbon-based magnetic materials have been proposed, i.e. graphene-based systems [5] and radical stacks [10]. In comparison to models of graphene-based systems, models of radical stacks exhibit a better way for designing carbon-based ferromagnets. Understanding the magnetic interaction between radicals is one of the most fundamental and important subjects for developing high-spin radical stacks. The topological principle proposed is in line with the McConnell rule, stating that directly overlapping $\pi$-spin is antiferromagnetically coupling. Thus homoradical stacks are likely to exhibit anti-parallel spin alignment and zero net collective spin. If a diamagnetic molecule is inserted between each pair of radicals in the stack, its spin polarization, albeit weak, can lead to an effective ferromagnetic ordering.

In this paper, in order to explore more about possibilities to design high-spin carbon-based magnetic materials, geometric structure, electronic structure and magnetic properties of alternating stacks of $\pi$-radical-halogenated-hydrocarbons and diamagnetic molecules have been investigated based on density-functional theory with dispersion correction. Moreover, in order to shed light on more about exchange coupling in alternating stacks, radicals and diamagnetic molecules with different ligand configurations have been employed to design stacks. Our calculated results demonstrate that the combination of molecules with different ligand configuration can form strong ferromagnetic stacks. These results would give some hints for designing new high-spin carbon-based ferromagnetic materials.

\section{COMPUTATIONAL METHOD}

All calculations have been performed by using the $\mathrm{DMol}^{3}$ code [11] with the double numerical basis sets plus polarization functional. For the exchange correlation terms, the generalized gradient approximation (GGA) PBE functional was used [12]. The TS dispersion-corrected method [13] was used to describe van der Waals interactions. The PBE-TS functional was successfully employed to predict the geometric structure and energy ordering of organic molecular crystal with high accuracy [14]. The all electron was used to describe the interaction between the core and valance electrons. The octupole expansion scheme was adopted for resolving the charge density and Coulombic potential. Moreover, the dipole slab correction was employed to accuracy exchange energy. The 
atomic charge and magnetic moment were obtained by using the Mulliken population analysis [15]. The real-space global cutoff radius was set to be $3.8 \AA$ for all atoms. The charge density is converged to $1 \times 10^{-6}$ a.u. in the self-consistent calculation. In the optimization process, the energy, energy gradient, and atomic displacement are converged to $1 \times 10^{-5}, 1 \times 10^{-4}$ and $1 \times 10^{-3}$ a.u., respectively. In order to determine the groundstate magnetic structure of each stack, we carried out total-energy calculations with full geometry optimization, allowing the relaxation of all atoms in stacks.

\section{RESULTS AND DISCUSSION}
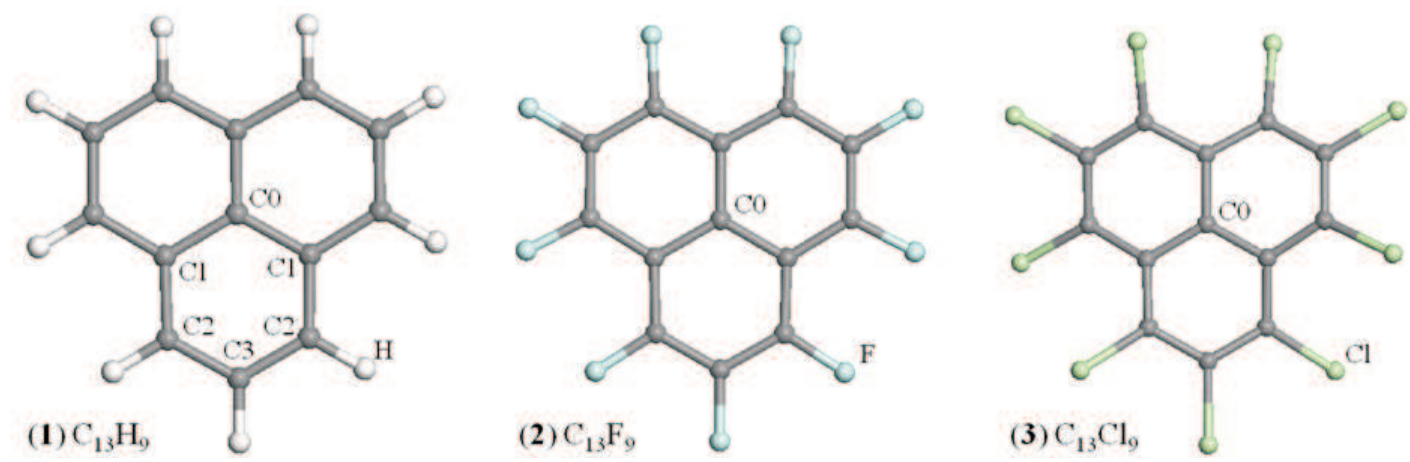

Fig. 1. The schematic geometric structure of radicals: (1) perinaphthenyl, (2) fluorinated perinaphthenyl, and (3) perchlorophenalenyl.

In this study, three radicals, i.e. perinaphthenyl (1), fluorinated perinaphthenyl (2), and perchlorophenalenyl (3) have been used in which radical (3) has been synthesized [16]. The schematic geometric structures of these three radicals are depicted in Fig. 1. As shown in Fig. 1, geometric structure of (1), (2) and (3) are similar. They have planar structure. Each of radicals $(\mathbf{1}),(\mathbf{2})$, and $(\mathbf{3})$ consists of three aromatic rings surrounded by nine $\mathrm{H}$, or $\mathrm{F}$, or $\mathrm{Cl}$ atoms, respectively, as shown in Fig. 1. All three radicals (1), (2), and (3) have $C_{3 v}$ symmetry with the $\mathrm{C}_{3}$ axis through their center carbon atom $(\mathrm{C} 0)$. Due to the $\mathrm{C}_{3 v}$ symmetry of (1), (2), and (3), C atoms can be classified in four sites C0, C1, C2, and C3, as labeled in Fig. 1. In each radical, there is one $\mathrm{C} 0$ site, three $\mathrm{C} 1$ sites, six $\mathrm{C} 2$ sites, and three C3 sites. Radicals (1), (2), and (3) have similarly atomic arrangement, however, their $\mathrm{C}$-C interatomic distances are slightly different due to the difference in boundary atoms of radicals, as shown in Table 1.

Radicals (1), (2), and (3) have one unpaired electron resulting in the total spin $S$ $=1 / 2$. The spin distribution of $(\mathbf{1}),(\mathbf{2})$, and $(\mathbf{3})$ is displayed in Fig. 2. Our calculated results show that the major spin of $(\mathbf{1}),(\mathbf{2})$, and $(\mathbf{3})$ delocalizes over all six C2 sites, as shown in Fig. 2. Besides, in radicals $(\mathbf{2})$ and $(\mathbf{3})$, there is small major-spin polarization on the $\mathrm{F}$ and $\mathrm{Cl}$ atoms. The minor spin of $(\mathbf{1}),(\mathbf{2})$, and $(\mathbf{3})$ is shared out for the $\mathrm{C} 1$ and C3 sites. These results demonstrate that unpaired electrons in radicals $(\mathbf{1}),(\mathbf{2})$, and (3) can delocalize nearly whole radicals. This feature is quite different from that of $3 d$ compounds, in which magnetic moment is mainly localized on $3 d$ atoms. 
Table 1. Some typical interatomic distances of radicals and stacks (in unit of $\AA$ ). $\mathrm{X}=\mathrm{H}, \mathrm{F}$, or $\mathrm{Cl}$.

\begin{tabular}{|l|l|l|l|l|l|l|}
\hline & C0-C1 & C1-C2 & C2-C3 & C1-C1 & C1-C2 & C3-X \\
\hline$(\mathbf{1})$ & 1.432 & 1.419 & 1.391 & 2.481 & 2.834 & 1.091 \\
\hline$(\mathbf{2})$ & 1.439 & 1.417 & 1.390 & 2.493 & 2.833 & 1.343 \\
\hline $\mathbf{3})$ & 1.440 & 1.429 & 1.401 & 2.493 & 2.833 & 1.725 \\
\hline $\mathbf{1 - A - 1}$ & 1.431 & 1.419 & 1.394 & 2.478 & 2.831 & 1.090 \\
\hline $\mathbf{1 - B - 1}$ & 1.431 & 1.420 & 1.394 & 2.479 & 2.832 & 1.091 \\
\hline $\mathbf{2 - A - 2}$ & 1.438 & 1.417 & 1.389 & 2.491 & 2.832 & 1.342 \\
\hline $\mathbf{2 - B - 2}$ & 1.438 & 1.418 & 1.390 & 2.491 & 2.833 & 1.343 \\
\hline $\mathbf{3 - A - 3}$ & 1.450 & 1.433 & 1.401 & 2.513 & 2.853 & 1.726 \\
\hline $\mathbf{3 - B - 3}$ & 1.449 & 1.433 & 1.401 & 2.510 & 2.851 & 1.726 \\
\hline
\end{tabular}
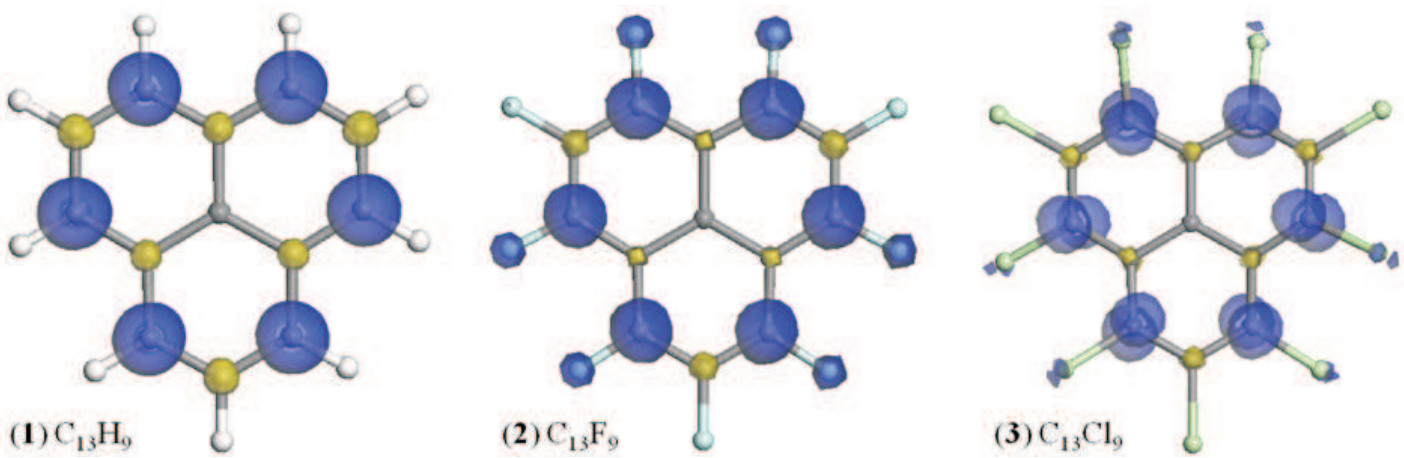

Fig. 2. The spin distribution of (1), (2) and (3). Color code: blue/dark (spin up), yellow/light (spin down). Density at the surface $=0.03 \mathrm{e} / \AA^{3}$.

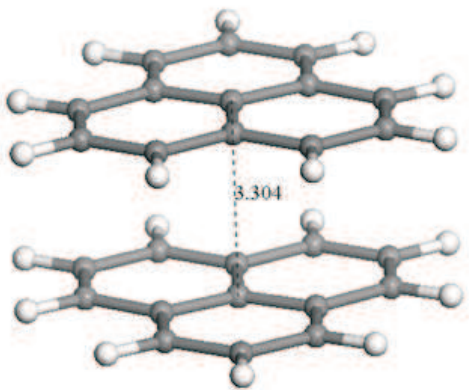

(1-1) $\left[\mathrm{C}_{13} \mathrm{H}_{9}\right]_{2}$

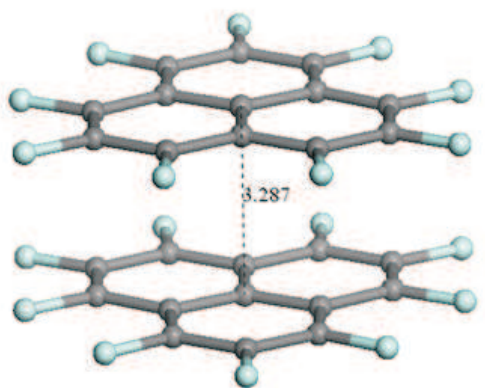

(2-2) $\left[\mathrm{C}_{13} \mathrm{~F}_{9}\right]_{2}$

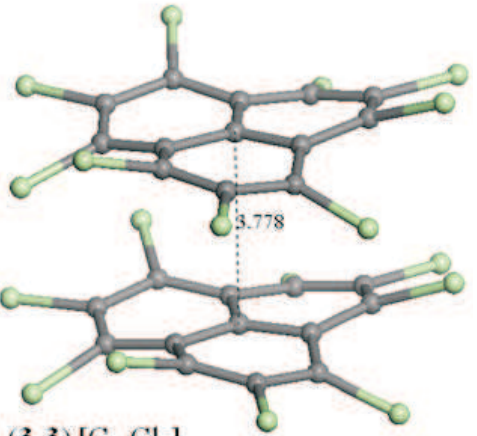

(3-3) $\left[\mathrm{C}_{13} \mathrm{Cl}_{9}\right]_{2}$

Fig. 3. The schematic geometric structure of dimers (1-1), (2-2) and (3-3).

Here, it is noted that, each radical has the total spin $S=1 / 2$. To design high spin molecules, radicals $(\mathbf{1}),(\mathbf{2})$, and $(\mathbf{3})$ have been used as building blocks. Our calculated results demonstrate that these radicals can form dimers (1-1), (2-2), and (3-3), as 
displayed in Fig. 3. Planar structure of $(\mathbf{1})$ and (2) is preserved in their dimeric form, as shown in Fig. 3. However, the planar structure of $(\mathbf{3})$ is not preserved in its dimer which can be attributed to strong Coulomb repulsion between large $\mathrm{Cl}^{-}$ions at the edge of $(\mathbf{3})$. The spacing between two radicals of $(\mathbf{3 - 3})$ obtained from our calculation is 3.778 $\AA$. This value is in good agreement with the previous experimental data of $3.78 \AA[16]$. This inter-radical distance is significantly larger than those of (1-1) and (2-2), only about $3.304 \AA$ and $3.287 \AA$, respectively.

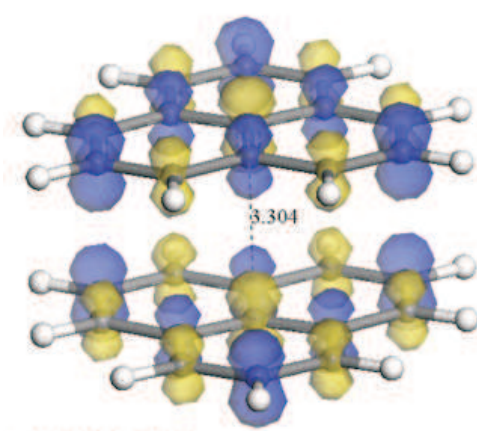

(1-1) $\left[\mathrm{C}_{13} \mathrm{H}_{0}\right]_{2}$

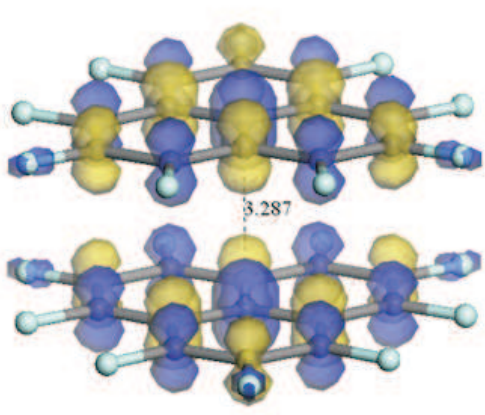

(2-2) $\left[\mathrm{C}_{13} \mathrm{~F}_{9}\right]_{2}$

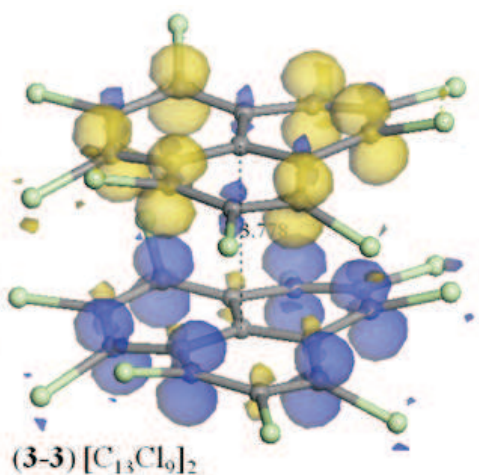

(3-3) $\left[\mathrm{C}_{13} \mathrm{Cl}_{0}\right]_{2}$

Fig. 4. The spin distribution of dimers (1-1), (2-2) and (3-3). Density at the surface $=0.03 \mathrm{e} / \AA^{3}$.

Our calculated results show that all dimers of radicals $(\mathbf{1}),(\mathbf{2})$, and $(\mathbf{3})$ have antiferromagnetic structure, as shown in Fig. 4. Fig. 4 shows that the magnetic structure of dimers (1-1) and (2-2) is similar, being different from that of dimer (3-3). In dimers (1-1) and (2-2), spin moment at each carbon atom is opposite to those of its nearest neighbors resulting in compensation of the total spin in each radical of dimers. This result can be attributed to strong face-to-face overlap between $\pi$ states of radicals of dimers (1-1) and (2-2). In dimer (3-3), distance between two radicals 3-3 is quite longer than those of dimers (1-1) and (2-2). Therefore, the overlap between $\pi$ orbitals of two radicals in dimer (3-3) is weaker in comparison to dimers (1-1) and (2-2). As a consequence, the hybridization between $\pi$ orbitals of two radicals in dimer (3-3) is not so strong. Hence, spin polarization in each radical of dimer (3-3) is not so much influent by another. In dimer (3-3), spin polarization is also mainly distributed on six C2 sites. This feature is similar to single radical (3). However, the total spin of dimer (3-3) is still compensated to zero due to direct face-to-face overlap between its two radicals $(\mathbf{3})$.

As presented above, our calculated results demonstrate that direct face-to-face overlap between planar radicals results in compensation of spin polarization. To avoid this problem, one approach is proposed, that is inserting a diamagnetic molecule between each pair of radicals to form alternating stacks. Moreover, to shed light more about exchange coupling in alternating stacks, diamagnetic molecules with different ligand configuration should be employed. Based on this approach, two diamagnetic aromatic rings, i.e. coronene $\mathrm{C}_{24} \mathrm{H}_{12}(\mathbf{A})$ and fluorinated coronene $\mathrm{C}_{24} \mathrm{~F}_{12}(\mathbf{B})$ were used as spacers between radicals. In this study, six alternating stacks have been designed, including $\mathbf{1 - A - 1}$, 


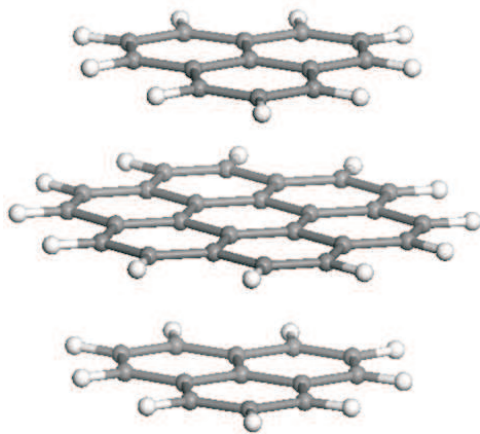

1-A-1

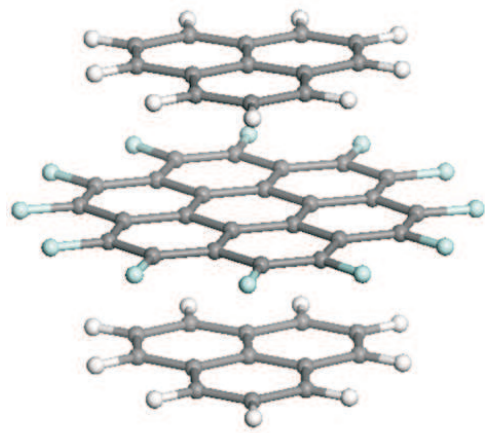

1-B-1

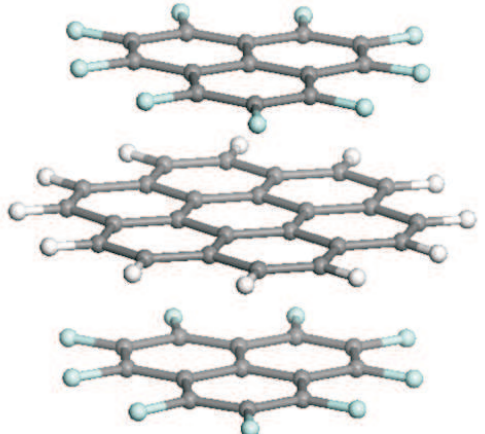

2-A-2

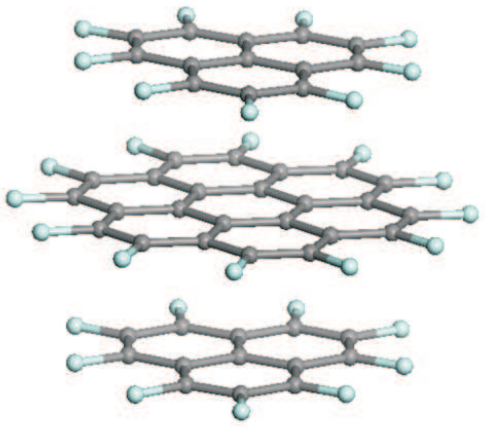

2-B-2

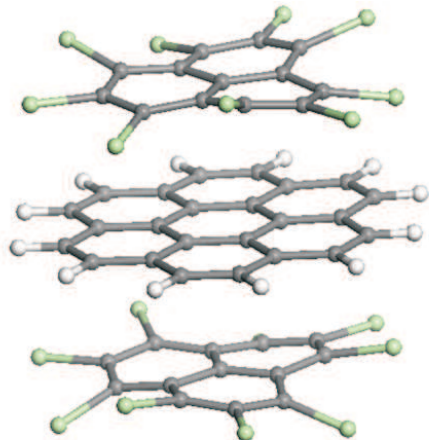

3-A-3

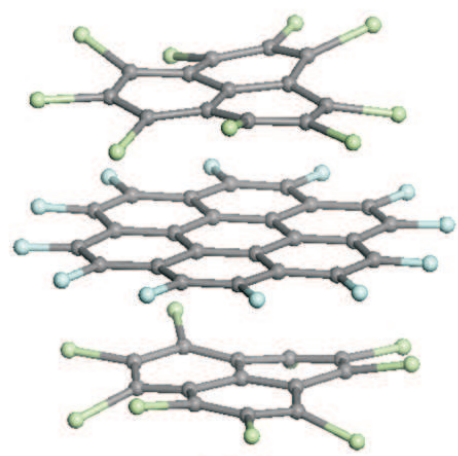

3-B-3

Fig. 5. Schematic geometric structure of alternating stacks, consisting of two radicals and a diamagnetic molecule in between.

1-B-1, 2-A-2, 2-B-2, 3-A-3, and 3-B-3. Schematic geometric structure of these stacks is presented in Fig. 5. The distance between the radical and diamagnetic molecule of these stacks is listed in Table 2.

Table 2. Some selected typical quantities of the stacks: Distance between radical and diamagnetic molecule (d); The effective spin-exchange (J); The SOMO energy gap $(\Delta E)$; The formation energy $\left(E_{f}\right)$.

\begin{tabular}{|l|l|l|l|l|l|l|}
\hline Stack & $\mathbf{1 - A - 1}$ & $\mathbf{1 - B - 1}$ & $\mathbf{2 - A - 2}$ & $\mathbf{2 - B - 2}$ & $\mathbf{3 - A - 3}$ & $\mathbf{3 - B - 3}$ \\
\hline$d(\AA)$ & 3.440 & 3.423 & 3.410 & 3.442 & 3.553 & 3.574 \\
\hline$J / k_{B}(\mathrm{~K})$ & 232 & 691 & 210 & 104 & 1073 & 2275 \\
\hline $\begin{array}{l}\Delta E_{S O M O} \\
(\mathrm{eV})\end{array}$ & 0.092 & 0.087 & 0.028 & 0.027 & 0.011 & 0.009 \\
\hline$E_{f}(\mathrm{eV})$ & -1.221 & -1.473 & -1.343 & -0.887 & -2.171 & -2.056 \\
\hline
\end{tabular}

As expected, all six alternating stacks avoid the typical antiferromagnetic spinexchange of indentical face-to-face radicals via spin polarization of a diamagnetic molecule 


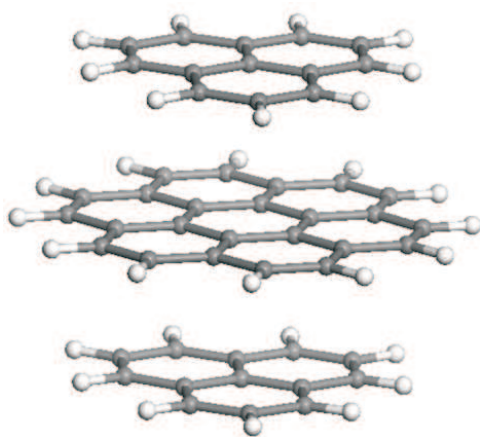

1-A-1

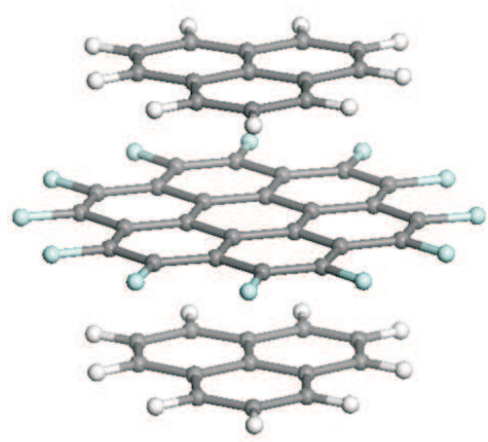

1-B-1

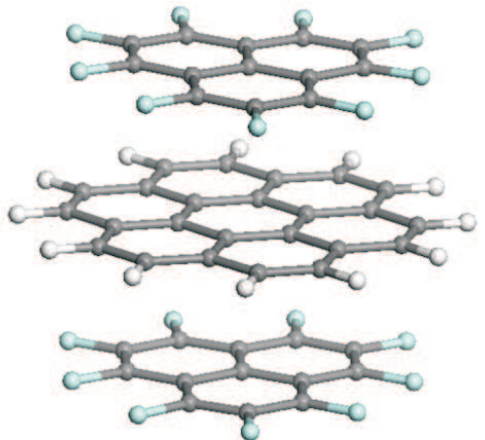

2-A-2

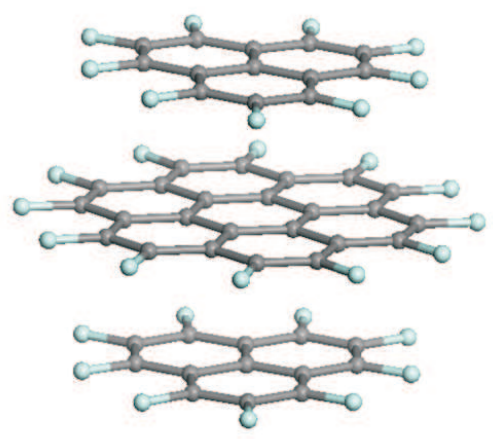

2-B-2

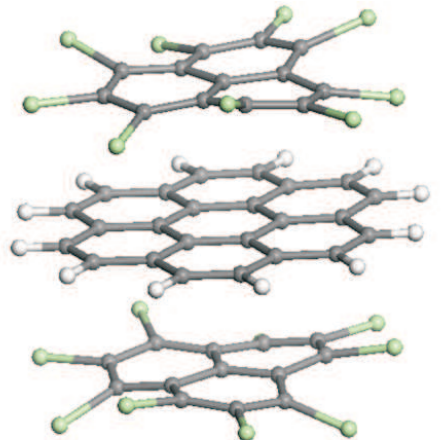

3-A-3

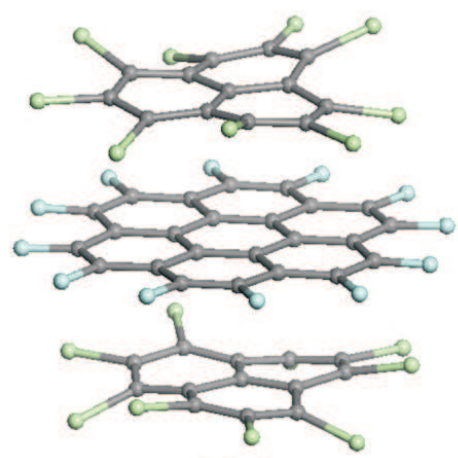

3-B-3

Fig. 6. The spin polarization in alternating stacks. Density at the surface $=0.03 \mathrm{e} / \AA^{3}$.

in between. Spin-exchange coupling of two radicals in each alternating stack is ferromagnetic resulting in the total spin $S=1$. The spin polarization in these alternating stacks is displayed in Fig. 6. Fig. 6 shows that the spin polarization in alternating stacks is mainly distributed on the $\mathrm{C} 2$ sites of two radicals. This feature is similar to single radical. To evaluate the stable of ferromagnetic structure of alternating stacks, the effective spin-exchange $J$ has been estimated by the singlet-triplet separation,

$$
J=\Delta E_{S T}=E_{S}-E_{T}
$$

The value of $J$ of alternating stacks is tabulated in Table 2. As shown in Table 2, the alternating stack 3-B-3 has the highest value of $J / k_{B}=2275 \mathrm{~K}$. This value is significant larger than those of the other alternating stacks. As we know, the strong $J$ usually corresponds to a small splitting of the two single occupied molecular orbitals (SOMOs) of alternating stacks. To confirm this, the splitting of the two SOMOs of alternating stacks has been calculated,

$$
\Delta E_{S O M O}=E_{S O M O 1}-E_{S O M O 2}
$$

Our calculated results confirm that the alternating stack $\mathbf{3}-\mathbf{B}-\mathbf{3}$ has the smallest value of $\Delta E_{S O M O}=0.009 \mathrm{eV}$. This value is significant smaller than those of other stacks, as listed in Table 2. A comparison between 1-A-1 and 1-B-1 stacks shows that the 
$J$ of $\mathbf{1 - B - 1}$ is significantly larger than that of $\mathbf{1 - A - 1}$. It is noted that, $\mathbf{1 - B - 1}$ is the combination of $\mathrm{C}_{13} \mathrm{H}_{9}(\mathbf{1})$ and $\mathrm{C}_{24} \mathrm{~F}_{12}(\mathbf{B})$ molecules with different ligand configurations, while 1-A-1 is the combination of $\mathrm{C}_{13} \mathrm{H}_{9}(\mathbf{1})$ and $\mathrm{C}_{24} \mathrm{H}_{12}(\mathbf{A})$ molecules with similar ligand configurations. These results demonstrate that the combination of molecules with different ligand configurations can enhance ferromagnetic coupling in stacks. A comparison between 2-A-2 and 2-B-2 stacks also confirms this.

To evaluate the stability of alternating stacks, their formation energy has been calculated by the following formula,

$$
E_{f}=E_{\text {stack }}-\left(2 E_{\text {radical }}+E_{\text {diamagnetic_molecule }}\right)
$$

where $E_{\text {stack }}, E_{\text {radical }}$, and $E_{\text {diamagnetic_molecule }}$ are the total electronic energy of stack, radical, and diamagnetic molecule, respectively. Our calculated results are listed on Table 2. The calculated formation energy of stacks is about from $-2 \mathrm{eV}$ to $-1 \mathrm{eV}$. It is noted that $1 \mathrm{eV}$ corresponds to temperature of about $10^{4} \mathrm{~K}$. These results show that all six stacks are predicted to be stable at room temperature.

\section{CONCLUSION}

In this paper, in order to explore a way to design high-spin carbon-based magnetic materials with strong ferromagnetic coupling, several forms of metal-free magnetic materials, i.e. single radicals, dimers of radicals, and alternating stacks of radicals and diamagnetic molecules have been investigated based on density-functional theory with dispersion correction and full geometry optimization. Radicals $\mathrm{C}_{13} \mathrm{X}_{9}$ (with $\mathrm{X}=\mathrm{H}, \mathrm{F}$, and $\mathrm{Cl}$ ) have spin of $1 / 2$. However, in their dimeric form, the net spin becomes zero due to antiferromagnetic spin-exchange which results from the direct $\pi-\pi$ overlap between radicals. To avoid the typical antiferromagnetic spin-exchange of identical face-to-face radicals, six alternating stacks of radicals and diamagnetic molecules with different ligand configurations have been designed. Our calculated results confirm that alternating stacks under consideration have ferromagnetic spin-exchange between radicals. In order to explore a way to tailor spin-exchange in stacks, the effect of ligand configuration on spin-exchange between radicals has been investigated. Our results show that spin-exchange interaction in stacks is significantly enhanced by using halogenated hydrocarbons of heavy atoms of halogen group. The combination of molecules with different ligand configuration can form strong ferromagnetic stacks. These results would give some hints for designing new high-spin carbon-based ferromagnetic materials.

\section{ACKNOWLEDGMENTS}

This research is funded by Vietnam National Foundation for Science and Technology Development (NAFOSTED) under grant number 103.01-2011.27. The computations presented in this study were performed at the Information Science Center of Japan Advanced Institute of Science and Technology.

\section{REFERENCES}

[1] S. K. Saha, M. Baskey, D. Majumdar, Adv. Mater. 22 (2010) 5531. 
[2] T. Makarova, F. Palacio, Carbon-Based Magnetism, Elsevier, Amsterdam, 2006.

[3] P. Esquinazi, A. Setzer, R. Höhne, C. Semmelhack, Y. Kopelevich, D. Spemann, T. Butz, B. Kohlstrunk, M. Lösche, Phys. Rev. B, 66 (2002) 024429.

[4] P. Esquinazi, D. Spemann, R. Höhne, A. Setzer, K.-H. Han, and T. Butz, Phys. Rev. Lett. 91 (2003) 227201.

[5] H. Xia, W. Li, Y. Song, X. Yang, X. Liu, M. Zhao, Y. Xia, C. Song, T. Wang, D. Zhu, J. Gong, Z. Zhu, Adv. Mater. 20 (2008) 4679.

[6] T. Enoki and K. Takai, Sol. State Commun. 149 (2009) 1144.

[7] S. Talapatra, et al., Phys. Rev. Lett. 95 (2005) 097201.

[8] A. V. Rode, et al., Phys. Rev. B 70 (2004) 054407.

[9] H. Ohldag, T. Tyliszczak, R. Höhne, D. Spemann, P. Esquinazi, M. Ungureanu, and T. Butz, Phys. Rev. Lett. 98 (2007) 187204.

[10] A. Ivanova, M. Baumgarten, S. Karabunarliev, and N. Tyutyulkov, Phys. Chem. Chem. Phys. 5 (2003) 4932.

[11] B. Delley, J. Chem. Phys. 92 (1990) 508.

[12] J. P. Perdew, K. Burke, M. Ernzerhof, Phys. Rev. Lett., 77 (1996) 3865.

[13] A. Tkatchenko and M. Scheffler, Phys. Rev. Lett., 102 (2009) 073005.

[14] A. Pedone, D. Presti, M. C. Menziani, Chem. Phys. Lett. 541 (2012) 12.

[15] R. S. Mulliken, J. Chem. Phys. 23 (1955) 1833. R. S. Mulliken, J. Chem. Phys., 23 (1955) 1841.

[16] P. A. Koutentis, R. C. Haddon, R. T. Oakley, A. W. Cordes and C. P. Brock, Acta Cryst., B57 (2001) 680. 